\title{
The effect of a temporary absence of tar- get velocity information on visual tracking
}

\author{
Patricia M. Cisarik \\ Southern College of Optometry \\ Frank E. Visco Jr. \\ Harold E. Bedell \\ Scott B. Stevenson \\ University of Houston College of \\ Optometry
}

\author{
Sanjeev Kasthurirangan \\ Abbott Medical Optics \\ Avesh Raghunandan \\ Ferris State University, Michigan Col- \\ lege of Optometry
}

\begin{abstract}
Experiments with the Rashbass 'step-ramp' paradigm have revealed that the initial catchup saccade that occurs near pursuit onset uses target velocity as well as position information in its programming. Information about both position and motion also influences smooth pursuit. To investigate the timing of velocity sampling near the initiation of saccades and smooth pursuit, we analyzed the eye movements made in nine 'step-ramp' conditions, produced by combining $-2,0$ and +2 deg steps with $-8,0$ and $+8 \mathrm{deg} / \mathrm{s}$ ramps. Each trial had either no temporal gap or a 50-ms gap during which the laser target was extinguished, beginning 25, 50, 75 or $100 \mathrm{~ms}$ after the step. Six subjects repeated each of the resulting 45 conditions 25 times. With no temporal gap, saccades were larger in the 'step-ramp-away' than the 'step-only' condition, confirming that saccade programming incorporates ramp velocity information. A temporal gap had no effect on the accuracy of saccades on 'step-only' trials, but often caused undershoots in 'step-ramp' trials. A 50-ms gap within the first $100 \mathrm{~ms}$ also increased the latency of the initial saccade. Although initial pursuit velocity was unaffected by a temporal gap, a gap that started at $25 \mathrm{~ms}$ reliably delayed pursuit onset for ramp motion of the target toward the fovea. Later gaps had a minimal effect on initial pursuit latency. The similar timing of the temporal gaps in target motion information that affect the initiation of saccades and pursuit provides further behavioral evidence that the two types of eye movements share pre-motor neural mechanisms.
\end{abstract}

Keywords: saccades, pursuit, velocity, latency, position error, visual gap

\section{Introduction}

A moving visual stimulus creates both retinal position and velocity errors, often resulting in a combination of saccadic and pursuit eye movements (Dodge, 1903). Early studies designed to characterize these eye movements identified position error as the fundamental stimulus for saccades and velocity error as the fundamental stimulus for pursuit (e.g. Rashbass, 1961, Lisberger, et al, 1987). However, more recent evidence indicates that retinal position and velocity errors both contribute to each type of eye movement (for reviews, see Krauzlis, 2005; de Xivry, \& Lefévre, 2007).

Specifically, several investigations showed that both position and velocity information affect the dynamics of a saccade to a moving stimulus (Gellman and Carl, 1991; Guan, et al, 2005; Keller and Johnsen, 1990; Newsome, et al, 1985). Analysis of saccades initiated by targets moving in simple ramp motion showed that saccades with longer latencies are more accurate than saccades with shorter latencies, suggesting that additional time for target motion processing refines the saccadic error signal (Gellman and Carl, 1991). By analyzing saccades to targets in ramp or double-ramp motion, Etchells et al. (2010) determined that the saccadic amplitude is influenced by velocity changes that occur up to 100 before saccadic onset. For targets that step in one direction and then undergo ramp motion in the opposite direction, both the direction and latency of the saccade are determined by the time the target crosses the fixation location, which 
Journal of Eye Movement Research $3(4): 4,1-16$
Cisarik, P., Kasthurirangan, S., Visco, F., Bedell, H., Stevenson, S., Raghunandun, A. (2010) The effect of a temporary absence of target velocity information on visual tracking must be calculated using both the target's velocity and position (Gellman and Carl, 1991). More recently, Guan et al (2005) found that the saccades made by monkeys to targets moving away from the fovea differ in peak velocity and duration from saccades of similar amplitude to targets moving towards the fovea. These results indicate that both retinal position error and target motion contribute to the saccade dynamics. Further, position and velocity information influence saccadic programming at different times. Using a 2D double 'step-ramp’ paradigm to present sudden target position and velocity changes, Schreiber et al. (2006) showed that the curved trajectory of the resulting saccades could be explained by the saccadic system's initial utilization of a position error, followed by the later incorporation of information conveyed by the velocity of the target's image.

Similarly, both position and velocity errors must be considered together to explain the amplitude of the saccades made by monkeys and cats during smooth pursuit (Keller and Johnsen, 1990; de Brouwer et al., 2001). When Keller and Johnson (1990) analyzed the saccades made by individual monkeys, they found a proportional increase in saccadic amplitude as both the target speed and its initial position error increased. Similar results were obtained by de Brouwer et al. (2002) during tracking of 'ramp-step-ramp' motion by human observers, for targets moving up to $15 \mathrm{deg} / \mathrm{s}$. These data contrast with those of an earlier study by Heywood and Churcher (1981), who reported that their human subjects added a small constant correction to the saccades made to moving targets without regard to the velocity of the target's motion.

Although smooth pursuit classically has been described as an oculomotor response to stimulus velocity (e.g. Rashbass, 1961, Lisberger, et al, 1987), an influence of position error also is evident. However, retinal position errors affect smooth pursuit velocity primarily when the pursuit system is already active and typically generate little or no pursuit if the eyes are directed toward a stationary target (Carl and Gellman, 1987; Morris and Lisberger, 1987). Blohm, Missal, and Lefevre (2005) used a 2dimensional stimulus presentation paradigm to introduce a position error with zero retinal image velocity orthogonal to the direction of ongoing pursuit. When a target was presented briefly to one side of the pursuit trajectory, smooth eye velocity in the direction of the transient target presentation was proportional to the size of the position error and independent of the characteristics of the ongoing pursuit. In addition to these behavioral studies, cells with parafoveal receptive fields in the nucleus of the optic tract (NOT) are modulated during smooth pursuit based on of the target's retinal position error, as well as its velocity, and acceleration (Das, et al, 2001).

During the latency period between the onset of target motion and the initiation of eye movement, the sensory information related to the position and velocity of a moving visual target is assimilated and transformed into the motor commands for saccadic and pursuit tracking. A disruption of sensory input during the latency period would be expected to influence the characteristics of the subsequent tracking movements. The principal goal of this study was to evaluate how the elimination of position and velocity information before the onset of tracking affects the timing and accuracy of saccadic and pursuit eye movements. In particular, by comparing the characteristics of saccades and pursuit to targets that disappeared briefly at various times before the start of tracking, we intended to elucidate how the pre-oculomotor neural mechanisms for saccadic and pursuit eye movements interact.

Well known is that the latencies for both saccades (Saslow, 1967; Fisher, \& Boch, 1983; Gezeck, \& Timmer, 1998) and pursuit (Krauzlis, \& Miles, 1996a, 1996b; Knox, 1996) are reduced if a temporal gap occurs between the offset of the fixation spot and a target's appearance, i.e. the "gap effect." These results suggest that a portion of the latency periods measured for saccades and pursuit with no temporal gap incorporates the time required to disengage fixation before an eye movement can take place. Although the position of a peripheral target can influence the amplitude of a subsequent saccade up to $80 \mathrm{~ms}$ prior to the saccadic onset (Becker and Jurgens,1979), to compensate accurately for a change in a target's position, the change must occur at least $140 \mathrm{~ms}$ before the onset of the saccade (Gellman and Carl, 1991). Churchland, et al. (2003) examined the effect of target disappearance on the initiation of smooth pursuit. Introduction of a 200-ms gap in target visibility just after the accelerating target reached full speed (i.e., between 100 and 150 ms after motion onset) caused a cessation of eye acceleration that began approximately $60 \mathrm{~ms}$ after the target disappeared, compared to the condition in which the moving target remained visible throughout the trial. Approximately $130 \mathrm{~ms}$ after the target disappeared, the 
Journal of Eye Movement Research $3(4): 4,1-16$
Cisarik, P., Kasthurirangan, S., Visco, F., Bedell, H., Stevenson, S., Raghunandun, A. (2010) The effect of a temporary absence of target velocity information on visual tracking velocity of pursuit began to fall. Eye acceleration decreased similarly when the target disappeared behind a visible occluder, but in this condition the later reduction of eye velocity was much less. Although these results show that a 200-ms gap in target visibility is sufficient to eliminate the drive signal for pursuit, still unknown is how the timing of a brief temporal gap in the visibility of target motion influences the latency and metrical properties of pursuit and saccades. In this study, we measured eye movements in response to the 'step-ramp' motion of a target that was extinguished for $50 \mathrm{~ms}$ at varying times shortly after the onset of its motion trajectory. We asked if this temporary loss of retinal velocity and position information prior to the initiation of tracking produces comparable perturbations in the timing and metrics of smooth pursuit and saccades.

\section{Methods}

\section{Subjects}

Six human subjects voluntarily participated in this experiment. All subjects had best-corrected visual acuity of 20/20 in each eye, no strabismus, and no known ocular or systemic pathology that could affect eye movements. The University of Houston Committee for the Protection of Human Subjects approved the procedures, and the subjects gave informed consent before participating in the study.

\section{Apparatus}

Eye movements (horizontal and vertical positions of each eye over time) were recorded at $120 \mathrm{~Hz}$ using a binocular dual-Purkinje image eye tracker and a Pentiumbased computer with 12-bit A-D converter. Prior to data collection, the target focus was adjusted to each subject's far point of accommodation, and target vergence was adjusted to the subject's far phoria position until no lateral or vertical movement was evident when the eyes were alternately covered. The data from the left eye were analyzed for 5 of the 6 subjects; the data from the right eye were analyzed for the remaining subject because the eye tracking was more reliable for that eye.

\section{Stimuli}

The stimulus was a red laser spot presented at the center of a dim, homogenous white background. The laser beam was reflected from a mirror mounted on a galva- nometer. A small deflection of the beam caused occlusion of the spot by a card interposed between the mirror and the screen. Otherwise, the laser spot was stationary on the projection screen. Movement of mirrors in the stimulator portion of the SRI dual-Purkinje eye tracker system produced the step / ramp motion trajectories.

\section{Procedure}

Before each trial, the subject fixated on the stationary stimulus located in the center of the visual field. On trial initiation, which was controlled by the subject, the fixation stimulus moved with one of nine motion patterns (see below) for $1000 \mathrm{~ms}$. Subsequently, the program extinguished the stimulus and then redisplayed the stimulus at the center of the field of view in preparation for the next trial. No temporal gap in stimulus visibility was presented between fixation offset and the onset of target motion.

The combination of each of the three horizontal target steps $(-2,0$, or $+2 \mathrm{deg})$ with each of the three horizontal ramp motions $(-8,0$, or $+8 \mathrm{deg} / \mathrm{s})$ produced the nine distinct motion patterns that were displayed. Specifically, each trial presented the stimulus with one of the following motion patterns: no motion (step $=0$; $\operatorname{ramp}=0$ ); 'ramp-only' (step = 0; ramp $=+/-8 \mathrm{deg} / \mathrm{s}$ ); 'step-only' (step = +/-2 deg; ramp =0); 'step-ramp-toward' the initial fixation position (step = +2 deg; ramp = -8 deg/s; step = $2 \mathrm{deg}$; ramp $=+8 \mathrm{deg} / \mathrm{s}$ ); or 'step-ramp-away' from the initial fixation position (step $=+2 \mathrm{deg}$; $\mathrm{ramp}=+8 \mathrm{deg} / \mathrm{s}$; step $=-2 \mathrm{deg}$; ramp $=-8 \mathrm{deg} / \mathrm{s}$ ). To compare the eye movements made to a continuously visible target to those made when the visibility of the target was briefly obscured, additional trials were generated in which a $50 \mathrm{~ms}$ gap in target visibility was inserted, beginning $25,50,75$, or $100 \mathrm{~ms}$ after the initiation of the target's motion. Thus, each block consisted of 45 trials (9 motion trajectories combined with 5 gap conditions), presented in a shuffled order, and each subject completed 25 blocks of trials.

The horizontal positions of the eyes in response to the displacement and motion of the laser spot were recorded for 1000 ms using a dual-Purkinje eye tracker (Crane, \& Steele-Applied Optics, 1985) and stored to a computer at a sampling rate of $120 \mathrm{~Hz}$. Trials were automatically rejected by the computer program if a blink occurred or if eye tracking was lost, and the trial condition subsequently was repeated. Differentiation of the raw data comprising the eye movement trace for each trial produced the eye 


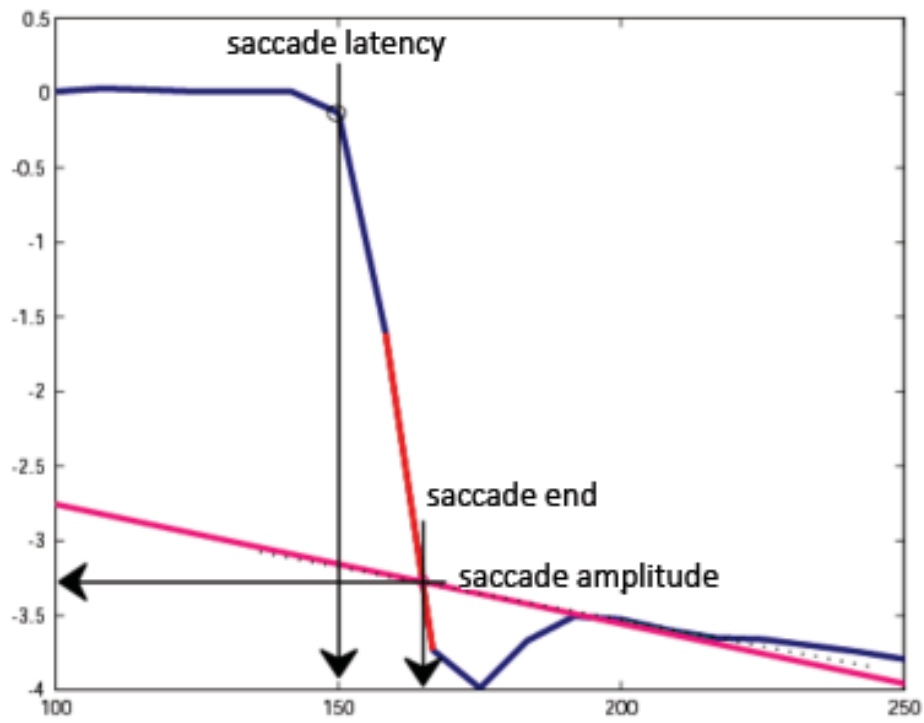

Figure 1: Determination of saccadic latency, amplitude, and endpoint. Saccade latency was determined as the point where eye velocity exceeded 20 degrees per second. Saccade amplitude and end time were determined by extrapolation to remove the dual-Purkinje eyetracker lens wobble artifact.

velocity trace for that trial. Analysis of the eye position and velocity traces established the saccade latency, pursuit latency, initial pursuit velocity, and post-saccadic position error for each trial. The saccade latency was defined as the length of time between the onset of the trial and the time when the eye velocity equaled or exceeded $20 \mathrm{deg} / \mathrm{s}$ (Figure 1). Pursuit latency was defined as the length of time between the onset of the trial and the achievement of an absolute eye velocity for three consecutive samples that was greater than or equal to three standard deviations of the smoothed eye velocity during trials with no ramp and no step. The initial pursuit velocity was defined as the average velocity of the de-saccaded eye position trace on ramp and step-ramp trials for the initial 50-ms interval after the onset of pursuit. The postsaccadic error was defined as the difference between the eye and target position traces at the end of the saccade. A comparison of these components across trials that varied in the temporal location of the gap was performed to evaluate the effect of a brief absence of velocity information on saccadic and pursuit programming.

\section{Results}

\section{Saccade analysis}

Figure 1 diagrams the method used to determine the initiation, amplitude, and endpoint of each saccade. As specified above, the initiation of a saccade was identified in the raw trace as the eye position sample at which the eye velocity first exceeded $20 \mathrm{deg} / \mathrm{s}$. Determining the amplitude of each saccade was complicated by the lenswobble artifact that appears in data collected from most subjects with the dual-Purkinje eye tracker (Deubel and Bridgeman, 1995). To circumvent the effects of lenswobble artifacts, the peak eye velocity during each saccade was estimated by drawing a line through the two adjacent samples in the trace that gave the steepest slope after the eye velocity exceeded $20 \mathrm{deg} / \mathrm{s}$ (the gray dashed line in Figure 1). To estimate the post-saccadic eye velocity, a second line was drawn through the 4th and 6th samples after the eye velocity fell below $20 \mathrm{deg} / \mathrm{sec}$ (the gray solid line in Figure 1). The selection of these two points in time (i.e., 33 and $50 \mathrm{~ms}$ after the end of the 


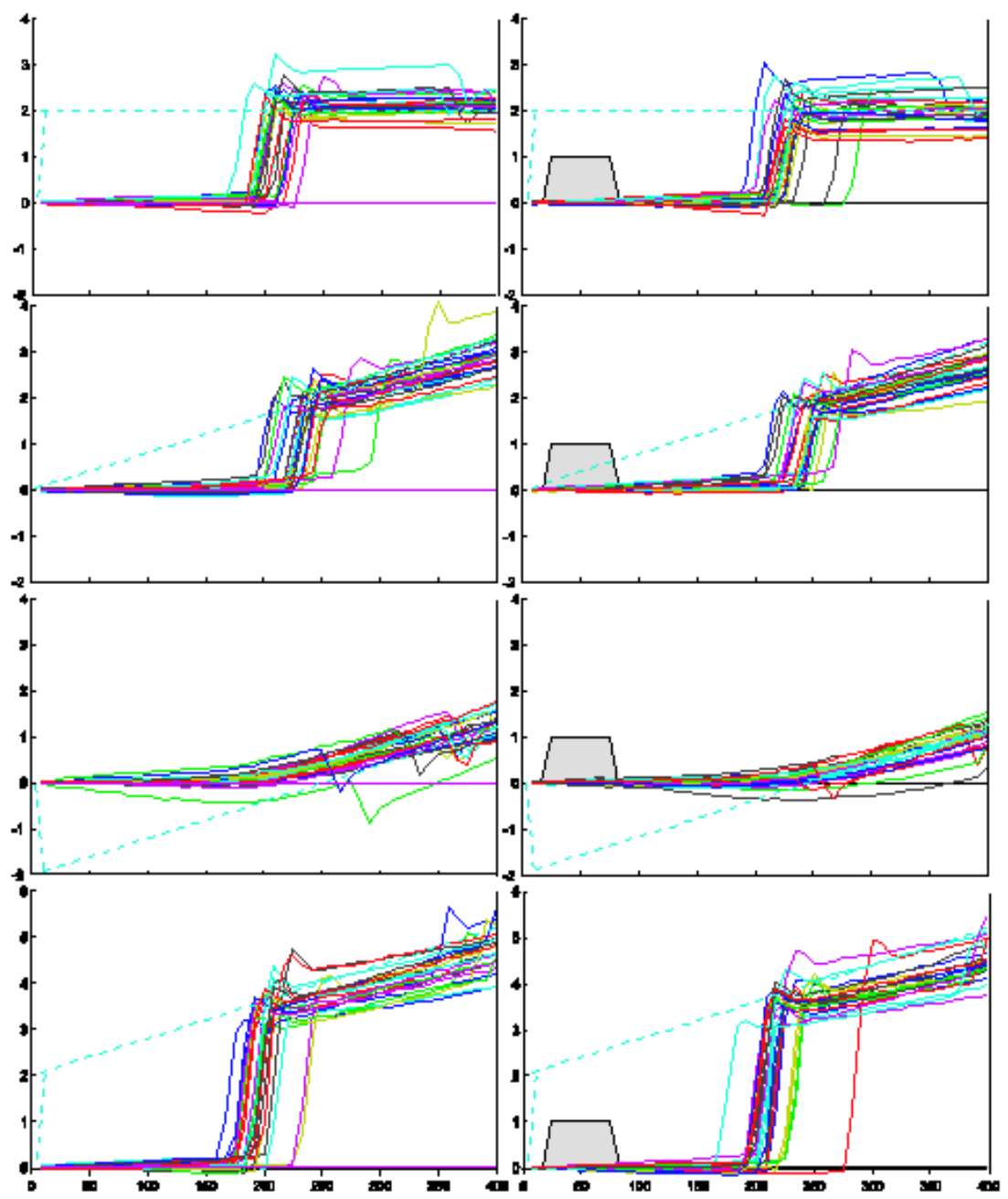

Figure 2: Raw eye movement traces for subject PMC, 8 of the 45 conditions. The dashed line plots target position; the gray box shows gap timing.

saccade was detected) was empirical, based on the duration of the subjects' saccades and the typical duration of the lens wobble artifact. Extending the second line leftwards until it intersected the first line provided an estimate of the endpoint of the saccade. The magnitude of the change in the eye position from the initiation of the sacade to the estimated saccadic endpoint at the intersection of the two lines delimited the saccade amplitude.

\section{Raw eye movement traces}

Figure 2 illustrates raw eye movement traces for subject PMC for eight of the 45 trial conditions tested. These traces are representative of the responses collected from the remaining subjects. The dashed line in each panel represents the trajectory of the target motion. The traces in the left panel present responses to target motion with continuous visibility. The traces in the right panel show responses to identical target trajectories with the interjectionof a 50 ms gap in target visibility starting 25 ms after initiation of target motion (indicated by the gray trapezoids). A comparison of the right and left panels in the bottom two rows shows that the onset of pursuit was delayed for this subject in the 'step-ramp-toward' condition, and the onset of saccades was delayed in the 'stepramp-away’ condition. 


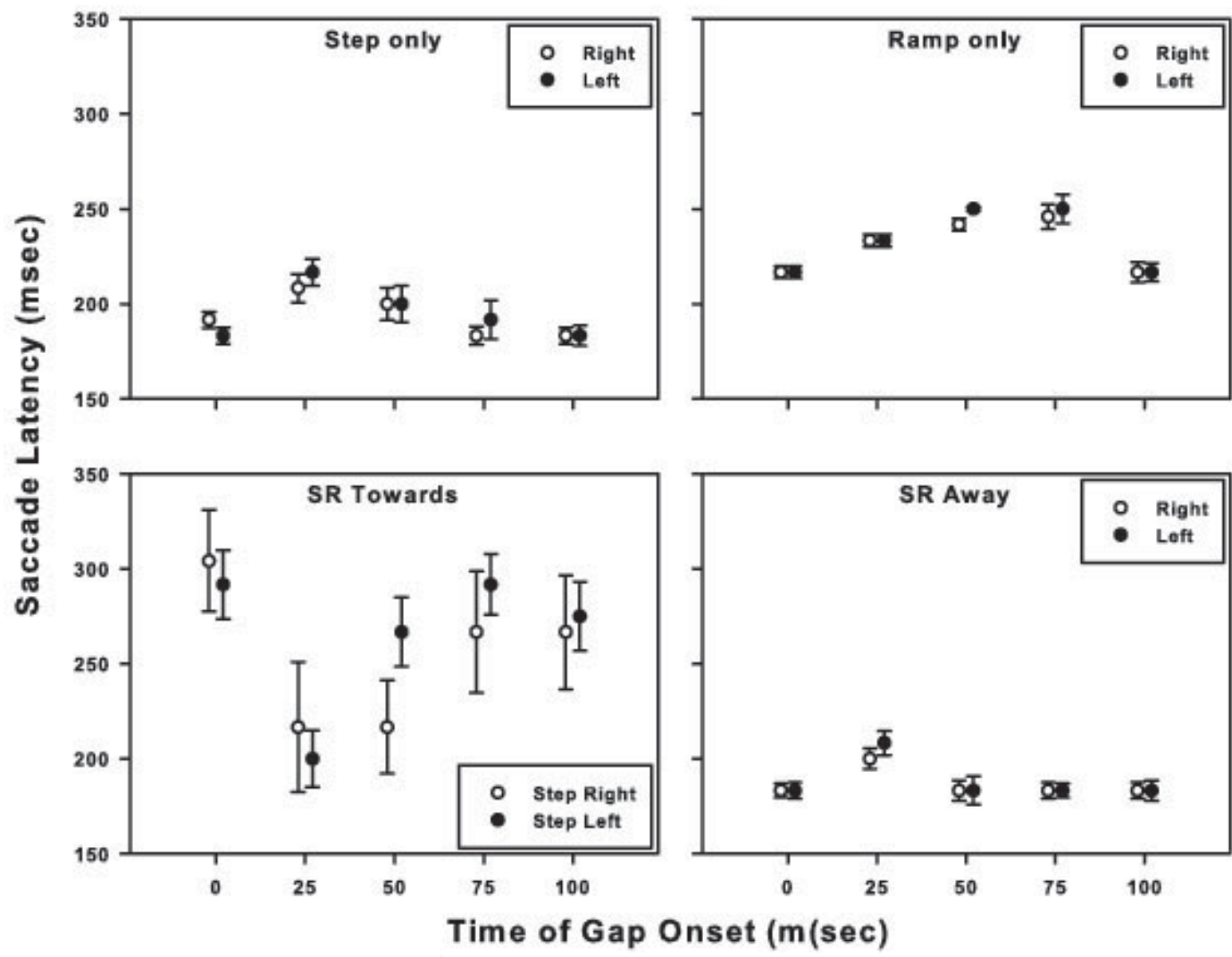

Figure 3: The eye position relative to the target position is plotted against the saccade latency after the gap, for all subjects, with each panel representing a different stimulus condition.

\section{Visual gap and saccade latencies}

The effect of the onset time of the gap in target visibility on saccade latencies is exhibited in Figure 3. Results for similar target motion trajectories (denoted in the title of each panel) in opposite directions are plotted together, e.g., 'step-only' target motion to the right and 'step-only' target motion to the left are plotted in the same panel. The dark symbols denote the results for initial target motion to the left; gray symbols denote the results for initial target motion to the right. The data for all subjects have been combined. Each symbol represents the mean of the saccade latencies for all trials for a given motion trajectory and gap condition. For example, the symbols with an abscissa value of $0 \mathrm{~ms}$ in each panel represent the mean saccade latencies for the motion trajectory designated in the panel title for trials in which there was no temporal gap in target visibility. Symbols with an abscissa value of $25 \mathrm{~ms}$ represent the mean sac- cade latencies for the motion trajectory specified in the panel title for trials in which a 50-ms temporal gap in target visibility began 25 ms after initiation of target motion, and so on. Except for the 'ramp-only' condition, the temporary absence of target visibility had the greatest effect on the saccade latency when the target disappeared $25 \mathrm{~ms}$ after the onset of target motion.

\section{Saccadic accuracy and latency}

Figure 4 illustrates the saccade landing site relative to the target position for subject AR on trials with 'steponly' (circles) and 'step-ramp-away' motion trajectories (diamonds). The target positions are indicated by the horizontal lines at \pm 2 deg for the 'step-only' trials and by the diagonal lines for the 'step-ramp-away' trials. Each panel presents the results for a different gap condition, with the time course of the gap indicated by the gray rectangle in each panel. For the 'step-only' trials, both with 

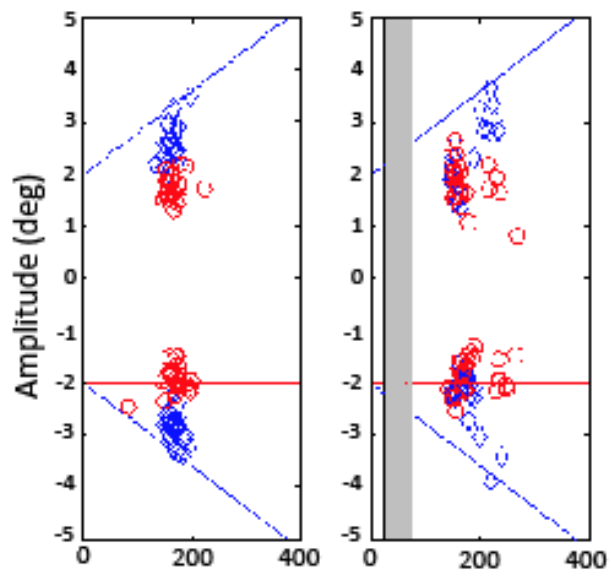

Cisarik, P., Kasthurirangan, S., Visco, F., Bedell, H., Stevenson, S., Raghunandun, A. (2010) The effect of a temporary absence of target velocity information on visual tracking

Figure 4: Saccade endpoint relative to the target position for subject AR for the step only conditions (red) and the stepramp away conditions (blue).

and without a gap in target visibility, the saccade landing sites cluster around the target position. Therefore, the accuracy of the saccades was not affected by a gap in the visibility of the target after the initial step. However, a temporal gap that begins 25 or 50 ms after the target step caused a delay in the onset of some of the saccades, as shown by the segregation of the data points for the 'steponly' condition into two clusters. In the 'step-rampaway' condition, temporal gaps that started at 25 and 50 ms resulted in reduced saccadic accuracy, as indicated by data points that fall farther from the diagonal line than in the 'no gap' condition, except for the trials during which the subject delayed his saccade.

The effects of a brief period of target invisibility on saccade accuracy are summarized in figure 5. Each panel shows the averaged data of all the subjects for a given motion trajectory, with the initial direction of motion indicated by the shading of the symbol. At the end of each saccade, the difference between the eye and target positions was measured. The mean of the trials for a given gap condition was determined and plotted against the gap condition. Consistent with the convention used in Figure 3, a value of $0 \mathrm{~ms}$ on the abscissa represents the 'no-gap' condition. The horizontal dashed line extending from zero on the ordinate indicates where the data point would be plotted if target position and eye position were coincident. A "lag" or a "lead" in eye position at the end of the saccade occurs when the distance between the eye position after the saccade and the previous fixation loca- tion is smaller or larger, respectively, than the distance between the target position and fixation.

For the target trajectories that contained both step and ramp motion, a gap in target visibility that began 25-50 ms after initiation of target motion was the most disruptive, causing an increase in saccadic latencies compared to targets with no gap in visibility. For the 'step-only' and 'ramp-only' conditions, saccade accuracy deteriorated further with later gap onsets, whereas for stepramp-toward and step-ramp-away conditions, later gaps resulted in some improvement in saccade accuracy. For the step-only and ramp-only conditions, trials with gap onsets later than 75-100 ms had a minimal effect on saccadic latencies (refer to fig. 3). However, the information available in the 40 - 100 ms after the target became visible again and before the saccade was made (i.e., saccade latency in figure 3 less the durations of the gap and target motion before the gap) was not of sufficient duration to improve the accuracy of the saccade, consistent with the findings of Gellman and Carl (1991).

For the step-only, ramp-only, and step-ramp-away conditions, gap onsets later than 25 - 50 ms produced saccades with longer latencies (with the increase being less than the duration of the gap), than in the no-gap and later-gap conditions, resulting in about 120-140 ms of target visibility after the gap and before saccade onset. Despite this longer target visibility saccades to targets with with early gaps were less accurate than saccades to 


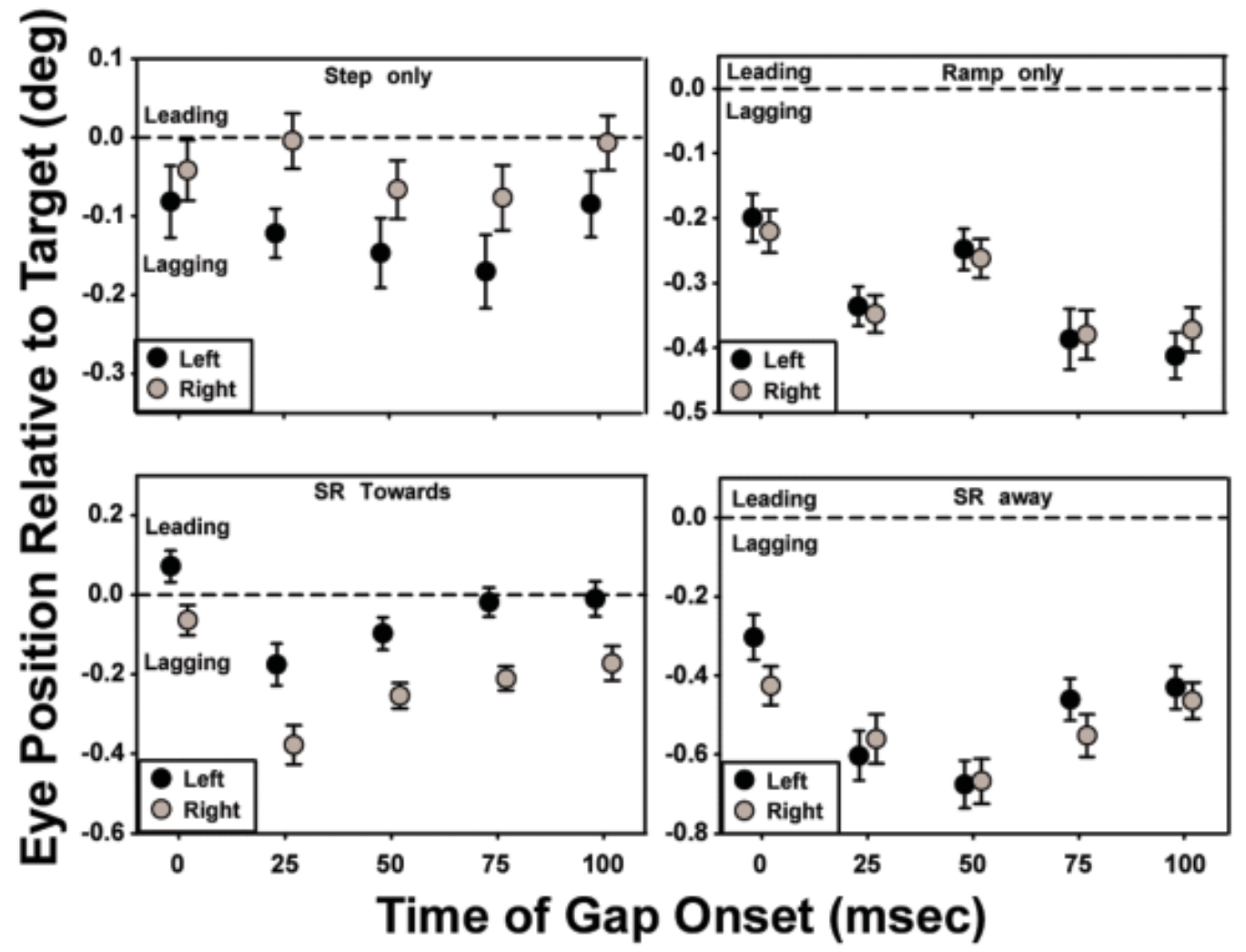

Figure 5: The effect of a gap on saccade accuracy: the gap is most disruptive when it onsets at 25-50 ms after initiation of target motion.

targets with later gaps. Saccade accuracy decreases with decreased target duration (Erlandson, \& Fleming, 1974); therefore, the position and velocity information available in the 120-140 ms after the return of target visibility for these early gap trials may have improved saccade accuracy from what it would have been had the target visibility not been restored, but not to the same degree as the accuracy for the later gap or no-gap trials.

To generate the scatter plots in Figure 6, the time from the initiation of target motion to the end of the gap time was subtracted from the saccade latency for each response. This value, which we call the gap-adjusted saccadic latency, was used as the independent variable. For example, if the saccade latency for a given trial was 300 ms and the trial condition contained a 50 ms gap in target visibility that began $25 \mathrm{~ms}$ after the onset of target motion, then the target would have reappeared $75 \mathrm{~ms}$ after the onset of the trial. The abscissa value used to plot this data point would be 225 (300 - 75) ms. The saccadic accuracy (eye position relative to target position) is plotted against the gap-adjusted saccade latency for all trials of all subjects, with the red data points representing the data for the no-gap condition and the gray data points representing the combined data for all of the gap conditions. The black line in each scatter plot designates the mean saccadic error for trials with a temporal gap across the gap-adjusted saccadic latencies. Frequency distributions of the saccadic latencies for the gap and no-gap conditions are included at the bottom of each panel. Except for the target motion conditions in which the step and ramp were in opposite directions, the distribution of saccadic latencies in the gap conditions was bimodal, with peaks occurring at approximately 75 and $150 \mathrm{~ms}$. For the 'ramp-only' and 'step-ramp-away' conditions with bimodal latency distributions, the mean saccadic error was less for trials with longer adjusted saccade latencies than for those with shorter adjusted saccade laten- 
Cisarik, P., Kasthurirangan, S., Visco, F., Bedell, H., Stevenson, S., Raghunandun, A. (2010) The effect of a temporary absence of target velocity information on visual tracking
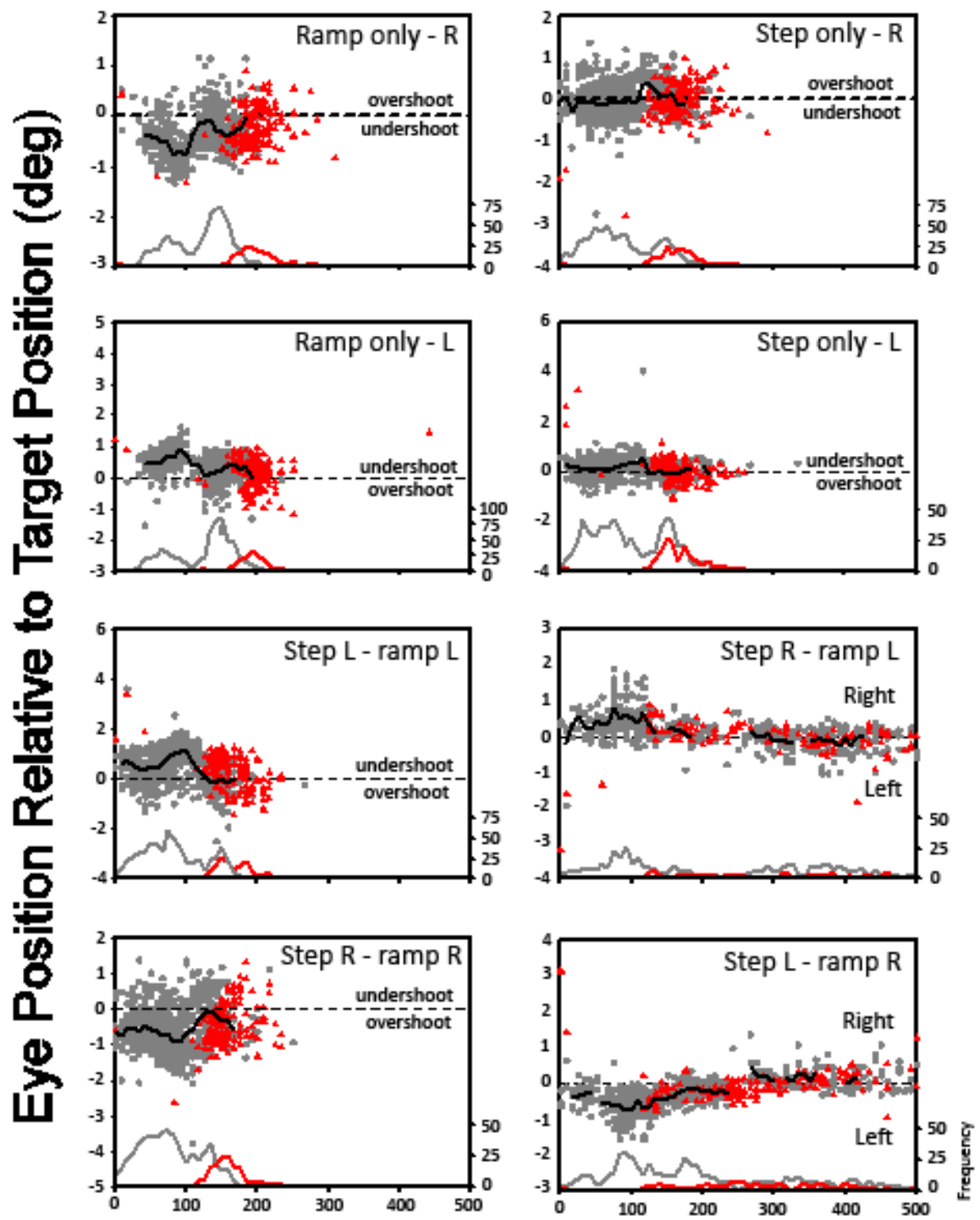

\section{Saccade Latency Relative to Gap (msec)}

Figure 6: Post-saccadic eye position relative to target position is plotted against saccade latency relative to the end of the gap. Data for all subjects are combined, with each panel representing a different step-ramp combination.

cies. That is, when subjects delayed the onset of their saccades, the resulting saccades were more accurate.

\section{Pursuit latency and velocity}

Figure 7 shows the effect of gap placement versus pursuit latency. The black symbols represent trials in which the initial direction of the target motion was to the left, and the gray symbols represent trials in which the initial direction of target motion was to the right. For the six conditions plotted, gaps in target-motion visibility that started 25 and $50 \mathrm{~ms}$ after initiation of target motion lead to an increase in pursuit latency relative to both the nogap condition and conditions with later gap onsets. 

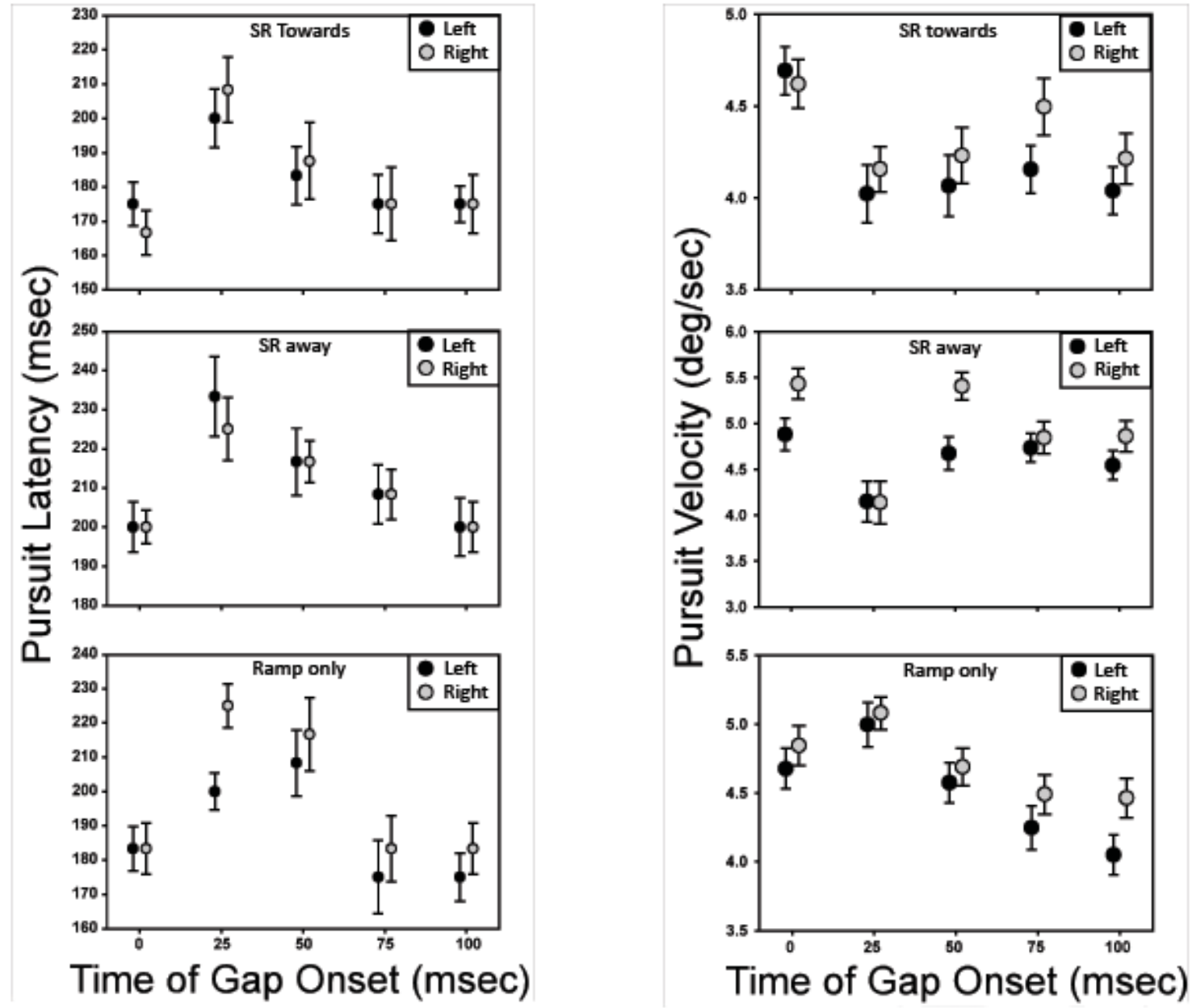

Figure 7 and Figure 8: Pursuit latency (left panel) and pursuit velocity (right panel) are plotted against the temporal location of the gap. The most disruptive gap onset is $25-50 \mathrm{~ms}$ after initiation of target motion.

Figure 8 illustrates how the onset of the gap affects the initial pursuit velocity. Again, each panel shows the averaged data for a given motion trajectory, with the initial motion direction indicated by the shading of the symbol (black - left; gray - right). For the 'ramp-only' conditions, the pursuit velocity is slightly elevated when the gap occurs at $25 \mathrm{~ms}$ and slowly declines for later gaps. The initial pursuit velocity in the 'step-ramp-towards' condition is reduced for all temporal gaps when compared to the no-gap condition. In the 'step-ramp-away' condition, only the gap at $25 \mathrm{~ms}$ causes a reduction in the pursuit velocity.

\section{Discussion}

The main contribution of this study relates to how a brief interruption of target visibility after the onset of target motion, but prior to the onset of eye movement, can influence subsequently executed saccades and pursuit. Because a component of velocity calculation is time, and because target velocity is reported to influence both pursuit and saccades (Etchells et al., 2010; Gellman and Carl, 1991; Keller and Johnsen, 1990; Morris and Lisberger, 1987), the goal of this study was to determine when during the initial, premotor assessment of visual target motion the absence of velocity information would be most disruptive. An interruption of target visibility

10 
Journal of Eye Movement Research $3(4): 4,1-16$
Cisarik, P., Kasthurirangan, S., Visco, F., Bedell, H., Stevenson, S., Raghunandun, A. (2010) The effect of a temporary absence of target velocity information on visual tracking
25-50 ms after the onset of target motion generally had the largest effect on the latency and accuracy of the ensuing saccade. In the ramp-only condition, an interruption of target visibility $25 \mathrm{~ms}$ after the onset of target motion had the greatest effect on pursuit latency and a lesser effect on initial pursuit velocity.

\section{Influence of target-velocity information on saccadic amplitude and latency}

Saccadic latency was relatively unaffected when target visibility remained uninterrupted for the first $75 \mathrm{~ms}$ of the trajectory for the 'step-only', 'step-ramp-away', and 'step-ramp-towards' conditions. In the 'ramp-only' condition, $100 \mathrm{~ms}$ of uninterrupted target visibility was required to produce saccadic latencies similar to those in the no-gap condition (figure 3). With the exception of the 'ramp-only' condition, saccadic accuracy was relatively unaffected when target visibility remained uninterrupted for the first $100 \mathrm{~ms}$ of the trajectory (figure 5). Using a double-step paradigm, researchers have shown that a change in target position prior to eye movements must occur within the first $80-100 \mathrm{~ms}$ of the target's trajectory if the saccade is to be accurate (Becker and Jurgens, 1979; Gellman and Carl, 1991). For a typical saccadic latency of $200 \mathrm{~ms}$, therefore, the target position that is sampled in the first $100 \mathrm{~ms}$ after the onset of target motion would provide less accurate information on trials with a temporal gap at 25 or 50 ms compared to no-gap trials, given the assumption that the saccadic system continues to sample the last seen location of the target throughout the temporal gap. Specifically, the saccadic lag would be expected to be greater than in the no-gap condition if the eye movement were made in response to the average target position during the first $100 \mathrm{~ms}$ after initiation of target motion. As indicated in the following analyses, the data from this study are not in accordance with this expectation.

The 'step-ramp-towards' condition with no temporal gap in target visibility elicited fewer saccades than did the other target-motion conditions, and the saccades that did occur had an average latency of approximately 300 ms (Figure 3, bottom left). With a ramp velocity of 8 $\mathrm{deg} / \mathrm{s}$, the stimuli in these trials passed through the initial fixation position $250 \mathrm{~ms}$ after target motion onset, or about $50 \mathrm{~ms}$ before the onset of the saccade. The long latencies of the saccades under this condition are consistent with the results of Rashbass (1961) and Gellman and Carl (1991), who showed that when the step was in the opposite direction of the ramp and of such magnitude that the target would cross its starting position approximately $200 \mathrm{~ms}$ after the ramp onset, saccades were typically either significantly delayed or cancelled. For this motion condition, the brief absence of target velocity information reduced saccadic latencies compared to the no-gap condition. Specifically, the introduction of a $50 \mathrm{~ms}$ gap in target visibility resulted in a reduction of the mean saccadic latency by approximately $90 \mathrm{~ms}$ when the gap started at $25 \mathrm{~ms}$ and by approximately $60 \mathrm{~ms}$ when the gap started at $50 \mathrm{~ms}$.

Because the ramp and step were in opposite directions in the 'step-ramp-towards' condition, short-latency saccades in the direction of the step lagged behind the moving target. Even so, the average saccadic lags in the 25and 50-ms gap conditions were only 0.2 and 0.3 deg, respectively. If the amplitude of these saccades had depended only on the target's retinal position error, then to produce the small lags observed in the 25 - and 50 -ms gap conditions, the position error of the target moving at 8 deg/s would need to have been sampled approximately 38 and $25 \mathrm{~ms}$, respectively, prior to the end of the saccadic eye movement. These values are much later than the estimates made previously for the end of the temporal sampling window for saccades, i.e. 80-100 ms before the start of a saccade (Becker and Jurgens, 1979; Gellman and Carl, 1991; Heywood and Churcher, 1981; Ron, et al, 1989; Findlay and Harris, 1993; Delinte et al, 2002). These data, therefore, are consistent with the conclusion reached by previous authors that the amplitude of the short-latency saccades in the 'step-ramp-towards' condition is computed using information about the target's velocity as well as its position (de Brouwer et al., 2002). However, although information about target velocity clearly contributes to the calculation of saccadic amplitude when a temporal gap occurs during the first 25 - 100 $\mathrm{ms}$ of the motion trajectory, this information apparently is not sufficient to cue the visual system that a saccade in the direction of the step is unnecessary. In contrast, the saccades in the 'step-ramp-towards' condition with no temporal gap generally occur after the ramp crosses the location of the target during fixation and, therefore, are in the direction of the ramp. On average, these saccades are accurate, which also is consistent with a contribution of velocity as well as position information to the calculation of saccadic amplitude. 
Journal of Eye Movement Research $3(4): 4,1-16$
Cisarik, P., Kasthurirangan, S., Visco, F., Bedell, H., Stevenson, S., Raghunandun, A. (2010) The effect of a temporary absence of target velocity information on visual tracking
The data from the 'ramp-only' and 'step-ramp-away' conditions also support the use of target-velocity information to improve the accuracy of saccadic programming. For example, in 'ramp-only' trials without a temporal gap, the eye position after the initial saccade lagged the target position, on average, by 0.2 deg (figure 5, top right panel). If the visual system used only the target's position to program the amplitude of saccades, then sampling would need to have occurred $25 \mathrm{~ms}$ before the end of the saccade, which is approximately $5 \mathrm{~ms}$ after saccade onset. Recently, Etchells et al. (2010) reported a similar result. The largest mean saccadic lag that we observed in all of the stimulus conditions is approximately $0.7 \mathrm{deg}$ ('step-ramp-away' condition with a 50 ms gap, Figure 5). Neglecting the 0.1 deg saccadic lag that we observed in the comparable 'step-only' condition, sampling would need to occur $85 \mathrm{~ms}$ before the end of the saccade (approximately $55 \mathrm{~ms}$ before the start of the saccade) if the amplitude of the saccade were based only on target position information. This worst-case estimate is still substantially smaller than the previous estimate of $80-100$ ms before the saccade for the end of position sampling by the saccadic system.

In the 'step-ramp-away' trials, the mean saccadic lag in the no-gap condition is larger than in the 'ramp-only' no-gap condition by $0.1-0.2 \mathrm{deg}$, which can be explained by the larger initial saccade required to reach the target ( 2 deg step +1.48 deg traveled during the $185 \mathrm{~ms}$ average latency period). Larger saccades take longer to complete, and saccadic programming may not account for the additional target distance traveled during this extra time. Additionally, larger saccades usually have larger undershoots, even in response to 'step-only' target motion. An analysis similar to that for the 'ramp-only' trials indicates that the visual system must use target velocity information to predict the target's future position and generate saccades with the observed degree of accuracy. For 'step-ramp-away' trials, only a gap that began $25 \mathrm{~ms}$ after the onset of target motion resulted in a prolonged saccadic latency, adding 20 - 25 ms compared to the nogap condition. The increase in the average lag at the end of the saccade in this condition is comparable to the distance that the target travels in 25 - 30 ms. When the onset of the temporal gap is $50 \mathrm{~ms}$ or later, the average saccadic latency is the same as in the no-gap condition, but saccadic amplitude remains reduced by $0.1-0.3$ deg compared to the no-gap condition. The larger saccadic lag compared to the no-gap condition suggests that the ab- sence of target-motion information starting 50 -100 ms after the target's onset impairs the visual system's ability to accurately predict the future location of the saccadic target, even though sufficient information is available to generate a saccade with a normal latency.

\section{Comparison with earlier results of experiments us- ing temporal gaps}

Some previous work investigated the effects of a temporal gap in target visibility on saccades and pursuit when the gap was introduced between the disappearance of the fixation target and the appearance of the saccadic or pursuit target, i.e., before any target-related visual information is available (Saslow, 1967; Fischer, \& Boch, 1983; Kingstone, \& Klein, 1993; Gezeck, \& Timmer, 1998; Kimmig, et al, 2002). Saccades and pursuit typically have shorter reaction times on trials with a temporal gap between fixation offset and target onset than on trials with no temporal gap (Saslow, 1967; Dorris, Pare and Munoz, 1997; Hunt, et al, 2004; Merrison and Carpenter, 1995; Knox, 1996; Krauzlis and Miles, 1996a,1996b; Kimmig et al, 2002). This 'gap effect' remains evident, although reduced, when the 'warning effect' of fixation offset is controlled by presenting an auditory signal just before each trial (Reuter-Lorenz, Oonk, Barnes and Hughes, 1995). A temporal gap between fixation and target motion onset could facilitate the onset of saccades and pursuit via a 'release' of fixation, brought about by a decrease in activity in the rostral superior colliculus (Dorris and Munoz, 1995; Munoz and Wurtz, 1993a, 1993b). Indeed, Krauzlis (2003) showed that shorter eye movement latencies in the gap conditions were associated with increased tonic and phasic firing rates of buildup neurons in the rostral superior colliculus when compared to the firing rates measured during trials with no temporal gap between fixation and target onset.

The "gap effect" must be considered as a possible explanation for the results of our study. Specifically, the subjects may have ignored the target motion before the temporal gap and perceived the absence of target visibility during the early part of the motion trajectory (i.e., starting at 25 or $50 \mathrm{~ms}$ ) simply as a temporal gap between the offset of fixation and the onset of target motion. If so, then a 50 ms gap in target visibility that begins $25 \mathrm{~ms}$ after the onset of target motion onset would be akin to a 75 ms gap between fixation offset and the start of target motion. Had this misperception occurred, then pursuit latencies for targets with gaps presented early in their 
Journal of Eye Movement Research $3(4): 4,1-16$
Cisarik, P., Kasthurirangan, S., Visco, F., Bedell, H., Stevenson, S., Raghunandun, A. (2010) The effect of a temporary absence of target velocity information on visual tracking trajectory should have had shorter latencies then their nogap counterparts. Figure 7, however, shows that the pursuit latencies for the 25 and 50 ms gap conditions were longer than those in the no-gap condition. Similarly, in the 'step-only', 'ramp-only', and 'step-ramp-away' conditions, a gap in target visibility between $25-75 \mathrm{~ms}$ also resulted in longer saccadic latencies relative to the no-gap condition (Figure 3).

If we assume that the visual system regards the first 75-100 ms of these conditions as a gap in target visibility that facilitates a 'release of fixation', then saccadic latencies should be measured from the end of the temporal gap rather than from the end of the fixation interval. Thus, to determine whether a "gap effect" occurs, a reduction of all of the latencies in the gap conditions in Figures 3 and 7 by $75-100 \mathrm{~ms}$ (the duration of the initial target motion plus the temporal gap) is necessary. The resulting adjusted latencies are 50-70 ms shorter than those in the nogap condition. This range of latency values is substantially longer than the 20 - 30 ms reduction found in the traditional "gap effect” paradigm (Saslow, 1967; Reulen, 1984a, b).

We might also consider that the oculomotor system responded to the stimulus that was visible before the temporal gap as if responding to a stimulus of very short duration. In a study to examine the effect of target duration on saccadic latency, van Loon and Adam (2006) found that saccadic latency decreased as target duration increased, from more than $260 \mathrm{~ms}$ for a target of $50 \mathrm{~ms}$ duration to approximately $200 \mathrm{~ms}$ for targets of longer duration. Erlandson and Fleming (1974) also found longer latencies for saccades made to targets of shorter duration. If the visual system responded to our gap trials as to saccadic stimuli of short duration, then a gap in target visibility that starts at 25 or 50 ms after target onset should produce longer latencies relative to the no-gap condition. Although our data did show an increase in saccadic latencies for the 25- and 50-ms gap conditions (Figure 3), the increases are much less than reported by van Loon and Adam for similar target durations. We presume that the information about target position and velocity that was obtained both before and after the target disappeared contributed to the shorter saccadic latencies in the present study, compared to those found by van Loon and Adam.

In 2008, Barnes and Collins showed that a long temporal gap (400 or $600 \mathrm{~ms}$ ) in target visibility has a more adverse effect on pursuit performance when introduced $50 \mathrm{~ms}$ after the initiation of target motion, compared to introduction later in the target's trajectory. They found that the eye velocity at the end of the extinction period more closely matched that of the reappearing target if the initial target exposure was for 100 ms compared to 50 $\mathrm{ms}$, but this difference between eye and target velocity did not decrease further for even longer exposure durations before the target disappeared. Barnes and Collins suggested these results indicate that an extraretinal (or internal drive) signal for pursuit evolves from sampling target velocity during approximately the first $150 \mathrm{~ms}$ of target motion. The results of the present study suggest that even a $50 \mathrm{~ms}$ interruption in target visibility during the first $100 \mathrm{~ms}$ of target motion delays the onset and perturbs the accuracy of the ensuing saccadic and pursuit eye movements if this interruption occurs early in the trajectory, i.e., 25 - $50 \mathrm{~ms}$ after the initiation of target motion. Introduction of a temporal gap reduces information about target velocity and position during the openloop period of visual processing. Without a commensurate increase in latency, which occurs for neither pursuit nor saccades with the 50 ms duration of extinction in our study, the ensuing eye movements must be initiated based on less information than when the target remains continuously visible.

A temporal gap between the offset of fixation and the subsequent onset of target motion has been shown to enhance the initial pursuit velocity in humans (Boman, \& Hotson, 1988) and in monkeys (Badler, \& Heinen, 2006). Our data show a reduction in pursuit velocity for the 25and 50-ms gap conditions compared to the no-gap condition when the target trajectory included a step (Figure 8). On the other hand, in the 'ramp-only' conditions, the earliest gap slightly enhanced the velocity of pursuit (by less than $0.5 \mathrm{deg} / \mathrm{s}$ ), which is consistent with the results of Badler, \& Heinen. The dissimilar effect of the 'stepramp' and the 'ramp-only' conditions on pursuit velocity may be attributable to a difference in the target's retinal location (central versus 2 deg peripheral) at the start of ramp motion. Alternatively, the process of de-saccading the eye movement traces in the 'step-ramp' trials may have artificially resulted in lower pursuit velocities in the 'step-ramp' conditions.

Processing of visual information for saccades and smooth pursuit 
Journal of Eye Movement Research $3(4): 4,1-16$
Cisarik, P., Kasthurirangan, S., Visco, F., Bedell, H., Stevenson, S., Raghunandun, A. (2010) The effect of a temporary absence of target velocity information on visual tracking
The evidence reported here that the same early temporal gap in target visibility exerts the greatest effect on the initiation of both saccades and pursuit is consistent with the suggestion that these two types of eye movements share some aspects of pre-motor neural processing (Newsome, et al., 1985; Krauzlis, \& Miles, 1998; Missal, et al., 2000; Missal, \& Keller, 2002; de Xivry, et al, 2006). Recent neurophysiological data suggest that pursuit and saccades also share neural mechanisms for response preparation. For example, the premotor nuclei in the brainstem that generate the burst of activity required to generate saccades receive input from both the saccadic and pursuit subregions of the frontal eye fields (Yan, et al, 2001). Also, Krauzlis (2004) found that rostral superior colliculus neurons of the monkey undergo a buildup of activity while previewing visual motion prior to pursuit initiation similar to that exhibited before saccades. Because this increased activity is not selective for the direction or speed of visual motion, Krauzlis suggested that visual motion signals can be used by these neurons to determine the goal for eye movements, regardless of whether the ensuing movements are pursuit or saccades. Krauzlis, Basso and Wurtz (2000) recorded from cells in the rostral superior colliculus of monkeys and found that fixation cells show the same directional preferences during pursuit as during small saccades. Krauzlis and Miles (1998) showed that microstimulation of sites in the oculomotor vermis initiated either pursuit-like or saccadelike eye movements, depending on the frequency of the stimulation. Additionally, Tagaki et al (1998, 2000) found that lesions to the same regions of the cerebellar vermis affected the immediate and adaptive control of both saccades and pursuit. Missal, et al (2000) studied the activity of burst neurons in the interstitial nucleus of Cajal in cats during both vertical saccades and vertical smooth pursuit and found that the activity was correlated with eye velocity for both types of eye movements. The authors proposed that these neurons may serve to integrate eye position and eye velocity signals prior to motor execution. Finally, Missal and Keller (2002) demonstrated that the stimulation of omnipause neurons in the pons of monkeys, which has been shown to interrupt saccades (Gandhi and Keller, 1999), also induces a strong deceleration of the eye during smooth pursuit. This result indicates that saccades and pursuit share pre-motor inhibitory neural mechanisms.

As orienting and tracking eye movements typically occur together when locating and following an object in the natural environment, coordination of saccadic and pursuit movements would be best achieved if the receipt of information about the temporal aspects of the target were simultaneous, or nearly so. The use of shared neural mechanisms right up to the point of decision-making about the type or types of eye movement that are required is not only spatially parsimonious with respect to brain tissue, but also provides a functionally efficient way to ensure that the information about target motion that arrives at the decision-making area(s) is consistent.

\section{Conclusion}

The initiation and accuracy of eye movements can be influenced by an interruption of target motion information during the pre-motor visual processing period. Generally, saccades to targets with a gap in visibility are more accurate if their initiation is delayed, suggesting that a minimum amount of velocity information must be processed for the goal of the eye movement (i.e., to reach the target) to be adequately calculated. The similarity of the temporal effects of target motion information on the initiation of saccades and pursuit is consistent with the emerging view that the two types of eye movements share pre-motor neural mechanisms, possibly up to the point where the decision about the eye movement type required to meet the goal is made. Additional behavioral and neurophysiological studies are needed to determine how the decision-making center uses the responses in these shared mechanisms to differentiate between the need to trigger a saccade and/or a pursuit movement.

\section{Acknowledgements}

Some of the results described here were presented as a poster at the 2004 meeting of the Vision Sciences Society. This study was supported in part by research grant, R01 EY12986 (to SBS), and by core center grant P30 EY07551 from the National Eye Institute, USA.

\section{References}

Badler, J.B., \& Heinen, S.J. (2006). Ancipatory movement timing using prediction and external cues. Journal of Neuroscience, 26(17), 4519-4525. 
Journal of Eye Movement Research $3(4): 4,1-16$
Cisarik, P., Kasthurirangan, S., Visco, F., Bedell, H., Stevenson, S., Raghunandun, A. (2010) The effect of a temporary absence of target velocity information on visual tracking
Becker, W., \& Jurgens, R. (1979). An analysis of the saccadic system by means of double step stimuli. $\mathrm{Vi}$ sion Research, 19, 967-983.

Blohm, G., Missal, M., \& Lefevre, P. (2005). Direct evidence for a position input to the smooth pursuit system. Journal of Neurophysiology, 94, 712-721.

Boman, D.K., \& Hotson, J.R. (1988). Stimulus conditions that enhance anticipatory slow eye movements. Vision Research, 28, 1157-1165.

Carl, J.R., \& Gellman, R.S. (1987). Human smooth pursuit, stimulus-dependent responses. Journal of Neurophysiology, 57, 1446-1463.

Churchland, M.M., Chou, I.H., \& Lisberger, S.G. (2003). Evidence for object permanence in the smooth-pursuit eye movements of monkeys. Journal of Neurophysiology, 90, 2205-2218.

Das, V.E., Economides, J.R., Ono, S., \& Mustari, M.J. (2001). Information processing by parafoveal cells in the primate nucleus of the optic tract. Experimental Brain Research, 140, 301-310.

De Xivry, J.O., Bennett, S.J., Lefevre, P., \& Barnes, G.R. (2006). Evidence for synergy between saccades and smooth pursuit during transient target disappearance. Journal of Neurophysiology, 95, 418-427.

De Xivry, J.O., \& Lefevre, P. (2007). Saccades and pursuit: two outcomes of a single sensorimotor process. Journal of Physiology, 584(1), 11-23.

Delinte, A., Gomez, C.M., Decostre, M.F., Crommelinck, M., \& Roucoux, A. (2002). Amplitude transition function of human express saccades. Neuroscience Research, 42(1), 21-34.

Deubel, H., \& Bridgeman, B. (1995). Fourth purkinje image signals reveal eye-lens deviations and retinal image distortions during saccades. Vision Research, 35(4), 529-538.

Dorris, M.C., \& Munoz, D.P. (1995). A neural correlate for the gap effect on saccadic reaction times in monkey. Journal of Neurophysiology, 73, 2558-2562.

Dorris, M.C., Pare, M., \& Munoz, D.P. (1997). Neuronal activity in monkey superior colliculus related to the initiation of saccadic eye movements. Journal of Neuroscience, 17, 8566-8579.

Erlandson, R.F., \& Fleming, D.G. (1974). Uncertainty sets associated with saccadic eye movements - basis of satisfaction control. Vision Research, 14, 481-486.
Etchells, P.J., Benton, C.P., Ludwig, C.J.H., \& Gilchrist, I.D. (2010). The target velocity integration function for saccades. Journal of Vision, 10(6:7), 1-14.

Findlay, J.M., \& Harris, L.R. (1993). Horizontal saccades to dichoptically presented targets of differing disparities. Vision Research, 33(7), 1001-1010.

Fischer, B., \& Boch, R. (1983). Saccadic eye movements after extremely short reaction times in the monkey. Brain Research, 260, 21-26.

Gandhi, N.J., \& Keller, E.L. (1999). Activity of the brain stem omnipause neurons during saccades perturbed by stimulation of the primate superior colliculus. Journal of Neurophysiology, 82(6), 3254-3267.

Gellman, R.S., \& Carl, J.R. (1991). Motion processing for saccadic eye movements in humans. Experimental Brain Research, 84(3), 660-667.

Gezeck, S., \& Timmer, J. (1998). Detecting multimodality in saccadic reaction time distributions in gap and overlap tasks. Biological Cybernetics, 78, 293-305.

Heywood, S., \& Churcher, J. (1981). Saccades to stepramp stimuli. Vision Research, 21(4), 479-490.

Hunt, A.R, Olk, B., von Muhlenen, A., \& Kingstone, A. (2004). Integration of competing saccade programs. Cognitive Brain Research, 19, 206-208.

Keller, E., \& Johnsen, S.D. (1990). Velocity prediction in corrective saccades during smooth eye movements in monkey. Experimental Brain Research, 80(3), 525531.

Kimmig, H., Biscaldi, M., Mutter, J., Doerr, J.P., \& Fischer, B. (2002). The initiation of smooth pursuit eye movements and saccades in normal subjects and in "express saccade makers". Experimental Brain Research, 144, 373-384.

Kingstone, A., \& Klein, R.M. (1993). Visual offsets facilitate saccadic latency: does predisengagement of visuospatial attention mediate this gap effect? Journal of Experimental Psychology: Human Perception and Performance, 19(6), 1251-1265.

Knox, P.C. (1996). The effect of the gap paradigm on the latency of human smooth pursuit eye movement. Neuroreport, 7, 3027-3030.

Krauzlis, R.J. (2003). Neuronal activity in the rostral superior colliculus related to the initiation of pursuit and saccade eye movements. Journal of Neuroscience, 23(10), 4333-4344. 
Journal of Eye Movement Research $3(4): 4,1-16$
Cisarik, P., Kasthurirangan, S., Visco, F., Bedell, H., Stevenson, S., Raghunandun, A. (2010) The effect of a temporary absence of target velocity information on visual tracking
Krauzlis, R.J. (2004). Activity of rostral superior colliculus neurons during passive and active viewing of motion. Journal of Neurophysiology, 92, 949-958.

Krauzlis, R.J. (2005). The control of voluntary eye movements, new perspectives. Neuroscientist, 11(2), 124-137.

Krauzlis, R.J., Basso, M.A., \& Wurtz, R.H. (2000). Discharge properties of neurons in the rostral superior colliculus of the monkey during smooth-pursuit eye movements. Journal of Neurophysiology, 84, 876891.

Krauzlis, R.J., \& Miles, F.A. (1996a). Decreases in the latency of smooth pursuit and saccadic eye movements produced by the "gap paradigm" in the monkey. Vision Research, 36, 1973-1985.

Krauzlis, R.J., \& Miles, F.A. (1996b). Initiation of saccades during fixation or pursuit: evidence in humans for a single mechanism. Journal of Neurophysiology, 76, 4175-4179.

Krauzlis, R.J., \& Miles, F.A. (1998). Role of the oculomotor vermis in generating pursuit and saccades: effects of microstimulation. Journal of Neurophysiology, 80, 2046-2062.

Lisberger, S.G., Morris, E.J., \& Tychsen, L. (1987). Visual motion processing and sensory-motor integration for smooth pursuit eye movements. Annual Review of Neuroscience, 10, 97-129.

Merrison, A.F.A., \& Carpenter, R.H.S. (1995). Express smooth pursuit. Vision Research, 35, 1459-1462.

Missal, M., de Brouwer, S., Lefevre, P., \& Olivier, E. (2000). Activity of mesencephalic vertical burst neurons during saccades and smooth pursuit. Journal of Neurophysiology, 83, 2080-2092.

Missal, M., \& Keller, E.L. (2002). Common inhibitory mechanism for saccades and smooth pursuit. Journal of Neurophysiology, 88, 1880-1892.

Morris, E.J., \& Lisberger, SG. (1987). Different responses to small visual errors during initiation and maintenance of smooth-pursuit eye movements in monkeys. Journal of Neurophysiology, 58(6), 13511369.

Munoz, D.P., \& Wurtz, R.H. (1993a). Fixation cells in monkey superior colliculus. I. Characteristics of cell discharge. Journal of Neurophysiology, 70, 559-575.
Munoz, D.P., \& Wurtz, R.H. (1993b). Fixation cells in monkey superior colliculus. II. Reversible activation and deactivation. Journal of Neurophysiology, 70, 576-589.

Newsome, W.T., Wurtz, R.H., Dursteler, M.R., \& Mikami, A. (1985). Deficits in visual motor processing following ibotenic acid lesions of the middle temporal visual area of the macaque monkey. Journal of Neuroscience, 5(3), 825-840.

Rashbass, C. (1961). The relationship between saccadic and smooth pursuit eye movements. Journal of Physiology, 159, 326-338.

Reulen, J.P.H. (1984a). Latency of visually evoked saccadic eye movements. I. Saccadic latency and the facilitation model. Biological Cybernetics, 50, 251-262.

Reulen, J.P.H. (1984b). Latency of visually evoked saccadic eye movements. II. Temporal properties of the facilitation mechanism. Biological Cybernetics, 50, 263-271.

Reuter-Lorenz, P.A., Oonk, H.M., Barnes, L.L., \& Huges, H.C. (1995). Effects of warning signals and fixation point offsets on the latencies of pro- versus antisaccades: implications for an interpretation of the gap effect. Experimental Brain Research, 103(2), 287293.

Saslow, M.G. (1967). Effects of components of displacement-step stimuli upon latency for saccadic eye movement. Journal of the Optical Society of America, 57, 1024-1029.

Tagaki, M., Zee, D.S., \& Tamargo, R.J. (1998). Effects of lesions of the oculomotor vermis on eye movements in primates: saccades. Journal of Neurophysiology, 80, 1911-1931.

Tagaki, M., Zee, D.S., \& Tamargo, R.J. (2000). Effects of lesions of the oculomotor vermis on eye movements in primates: smooth pursuit. Journal of Neurophysiology, 83, 2047-2062.

Van Loon, E.M., \& Adam, J.J. (2006). Saccadic latency as a function of target duration in a spatial localization task. Perceptual and Motor Skills, 102(1), 165170 .

Yan, Y.J., Cui, D.M., \& Lynch, J.C. (2001). Overlap of saccadic and pursuit eye movement systems in the brain stem reticular formation. Journal of Neurophysiology, 86, 3056-3060. 